Research Article

\title{
Characterization of Dielectric Relaxation Process by Impedance Spectroscopy for Polymers: Nitrile Butadiene Rubber and Ethylene Propylene Diene Monomer
}

\author{
Jae Kap Jung $\mathbb{D}^{1},{ }^{1}$ Young Il Moon, ${ }^{2}$ Gyung Hyun Kim, ${ }^{3}$ and Nae Hyung Tak ${ }^{1}$ \\ ${ }^{1}$ Center for Energy Materials Metrology, Korea Research Institute of Standards and Science, Daejeon 34113, Republic of Korea \\ ${ }^{2}$ Department of Electrical Engineering, Pohang University of Science and Technology, Pohang 37673, Republic of Korea \\ ${ }^{3}$ Department of Physics and Research Institute of Natural Science, Gyeongsang National University, Jinju 52828, \\ Republic of Korea
}

Correspondence should be addressed to Jae Kap Jung; jkjung@kriss.re.kr

Received 29 June 2020; Revised 8 November 2020; Accepted 11 November 2020; Published 7 December 2020

Academic Editor: Carlos Palacio

Copyright (C) 2020 Jae Kap Jung et al. This is an open access article distributed under the Creative Commons Attribution License, which permits unrestricted use, distribution, and reproduction in any medium, provided the original work is properly cited.

\begin{abstract}
We invented a dispersion analysis program that analyzes the relaxation processes from dielectric permittivity based on a combination of the Havriliak-Negami and conductivity contribution functions. By applying the created program to polymers such as nitrile butadiene rubber (NBR) and ethylene propylene diene monomer (EPDM), several relaxation processes were characterized: an $\alpha$ process due to segmental motions of the $\mathrm{C}-\mathrm{C}$ bond, an $\alpha^{\prime}$ process attributed to fluctuations in the end-to-end dipole vector of the polymer chain, the conduction contribution by the filler observed above room temperature, and secondary relaxation processes $\beta$ and $\gamma$ of motion for the side group in NBR. In the EPDM specimen, the $\beta$ process associated with the rotational motion of the side groups, the $\alpha$ process associated with the relaxation of local segmental motion, and the $\alpha \beta$ process associated with the origin of the $\beta$ process at high temperatures above $305 \mathrm{~K}$ were observed. The Maxwell-Wagner-Sillars effect and conduction contribution were also presented. The molecular chains responsible for the relaxation processes were assigned by building molecular models of the two polymers. The temperature dependence of the relaxation strength and the shape parameters that characterize the process were investigated. From the temperaturedependent relaxation analysis, the merged $\alpha \beta$ process, activation energy, and glass transition temperature were determined and compared.
\end{abstract}

\section{Introduction}

Many kinds of rubbery polymers are used in O-rings for hydrogen gas sealing under high-pressure hydrogen infrastructure conditions; nitrile butadiene rubber (NBR), ethylene propylene diene monomer (EPDM), and fluoroelastomer (FKM) rubbers are used as sealants and gaskets in valves and pipelines [1-6]. Previous studies have investigated physical properties such as the glass transition, volume swelling, and thermal stability, mechanical properties such as tensile strength, compression, elongation, and shear behavior at high hydrostatic pressure, and transport properties such as permeability, diffusivity, and solubility of hydrogen.
Dielectric spectroscopy provides a means to characterize the dielectric and electrical behaviors of polymers and has been widely employed to investigate the nature of molecular motion and related relaxation behaviors [7-13]. The dielectric response is usually controlled by $\alpha$ relaxation, whose relaxation time steeply increases when the temperature decreases toward the glass transition temperature, $T_{\mathrm{g}}$. The $\alpha$ process corresponds to the segmental motions of the main chains. In addition to $\alpha$ relaxation, additional relaxations called secondary relaxations or $\beta$ relaxations can be active on faster time scales [14-17].

Only a few dielectric studies have been performed on NBR. Dielectric relaxation spectroscopy and the dynamic mechanical analysis of NBR composites have identified $\alpha$ 
and $\alpha^{\prime}$ relaxation processes. The observed phenomena and their temperature dependence have been investigated in view of the molecular dynamics of the chains, filler effects, and glass transition [18]. The observed relaxations follow the Havriliak-Negami (HN) approach for the distribution of relaxation times. However, relaxations related to conductivity and secondary relaxations have not been detected in NBR. The $\alpha$ relaxation peaks of EPDM have been detected in the graph of dielectric $\tan \delta$ data versus temperature, where the height and position of the peaks changed with the thermal aging time [19]. The $\alpha$ process for neat EPDM nanocomposites was observed only in the measurement of the imaginary dielectric permittivity versus the frequency, in which the dielectric spectra were well fitted by the HN permittivity function. However, the $\beta$ relaxation peak and other relaxations were not detected [20].

Research on dielectric spectroscopy has been performed with limited observations of relaxation processes and has not revealed all the relaxations embedded in polymers. The interfacial or Maxwell-Wagner-Sillars (MWS) relaxation process occurs in heterogeneous systems, including the conduction contribution in two polymers, which has not been investigated until now. Moreover, relatively less attention has been given to the investigation of the dielectric and electrical behaviors of polymers, especially at temperatures higher than the glass transition temperature $T_{\mathrm{g}}$. The dielectric spectrum is mainly governed by the relaxation time for relaxation processes in polymers. This spectrum occurs over a wide frequency range due to line broadening from distributions in the relaxation times and the superposition of corresponding relaxation processes [21, 22]. Therefore, it is difficult to fully discern and characterize the overall relaxation processes embedded in the studied polymers.

This work decomposes the superimposed dielectric spectrum and assigns the peaks of the relaxation processes in the dielectric spectrum, resulting in the characterization of several relaxation processes with a developed dispersion analysis program [23]. The upgraded algorithm is used to analyze the relaxation processes by selectively satisfying either real or imaginary dielectric data based on three governing functions - the Cole and Cole [24], Davidson and Cole [25], and HN [26] functions-together with the conduction contribution. The upgraded program is applied to characterize the entire relaxation processes that exist in the two polymers-NBR and EPDM-under study. As the extension of dielectric spectroscopy research on NBR was investigated recently [23], research on EFDM has been completed. The present work was performed for NBR specimens produced from both different batches and compositions in [23]. Furthermore, this research is extended to lower frequencies reaching $0.01 \mathrm{~Hz}$. These investigated results for both polymers are discussed and reviewed. The molecular chains of the two polymers responsible for the relaxation processes are assigned by molecular modeling. The temperature dependence of the relaxation strength and shape parameters for the relaxation spectra are investigated. Upon finding the $\alpha$ and $\beta$ relaxations, conduction levels and MWS contributions in the two polymers, the activation energy, and glass transition temperature are obtained and discussed. The origin of the merged $\alpha \beta$ process in EPDM was first revealed from the temperature dependence of the relaxation spectra and relaxation strength.

\section{Materials and Methods}

2.1. Sample Preparation. NBR is a butadiene-based synthetic rubber made by copolymerizing combinations of butadiene $\left(\mathrm{CH}_{2} \mathrm{CH}=\mathrm{CHCH}_{2}\right)$ and acrylonitrile $\left(\mathrm{CH}_{2} \mathrm{CHCN}\right)$. The NBR specimen used in this work was synthesized by a Korean domestic company and contained 50\% carbon black as a filler. The acrylonitrile content amounts to $34 \%$ of the total amount of NBR. The glass transition temperature varies depending on the acrylonitrile content. NBR is widely used as a sealing material due to its excellent gas resistance [27]. Thus, NBR polymer materials have been used as O-ring seals for flange connections, threaded connectors, and various valves in high-pressure hydrogen infrastructures.

EPDM is an ethylene-based synthetic rubber made by copolymerizing various combinations of ethylene $\left(\mathrm{CH}_{2} \mathrm{CH}_{2}\right)$, propylene $\left(\mathrm{CH}_{2} \mathrm{CHCH}_{3}\right)$, and ENB monomer. EPDM elastomers have excellent heat, ozone, weathering, and aging resistance [28]. EPDMs can be used in a wide range of applications, and typical applications include radiators, heater hoses, O-rings, and gaskets. The EPDM specimen used herein was also synthesized by a domestic Korean company, and $34 \%$ carbon black was included as a filler during the fabrication of the EPDM specimen. The chemical compositions of both the NBR and EPDM specimens are summarized in Table 1 [29].

2.2. Impedance Spectroscopy System. The impedance spectroscopy system used to measure the complex dielectric permittivity (Figure 1) is composed of a temperature-controlled chamber, a digital multimeter (Fluke $8842 \mathrm{~A}$ ) for temperature monitoring, and an impedance analyzer (VSP-300) with a GPIB interface to a PC. The temperature is controlled and maintained by a heater and a circulating refrigerating medium varying at an interval of $5 \mathrm{~K}$ with temperature stability better than $0.2 \mathrm{~K}$ in a temperature range of $233 \mathrm{~K}$ to $404 \mathrm{~K}$. The discshaped specimens $(50 \mathrm{~mm}$ in diameter and $2.4 \mathrm{~mm}$ in thickness) are placed between two copper electrodes for this measurement.

Both the real and imaginary permittivities from the measurements using the impedance analyzer are calculated with the following relation:

$$
\begin{aligned}
& \varepsilon^{\prime}=\frac{d}{\omega \varepsilon_{0} A} \frac{Z^{\prime \prime}}{\left(Z^{\prime 2}+Z^{\prime \prime 2}\right)}, \\
& \varepsilon^{\prime \prime}=\frac{d}{\omega \varepsilon_{0} A} \frac{Z^{\prime}}{\left(Z^{\prime 2}+Z^{\prime \prime 2}\right)},
\end{aligned}
$$

where impedance $Z=Z^{\prime}-j Z^{\prime \prime}$ and $Z^{\prime}$ and $Z^{\prime \prime}$ are the real and imaginary impedance values, respectively. $A$ is the area of the specimen, and $d$ is the thickness of the cylindrically 
TABLE 1: Chemical compositions of NBR and EPDM specimens.

\begin{tabular}{lcc}
\hline Function & NBR & EPDM \\
\hline Polymer & NBR (40)* & EPDM (58) \\
Filler-reinforcing & Carbon black (50) & Carbon black (34) \\
Processing aid & 1, 2-benzenedicarboxylic acid (6) & - \\
Antioxidant & 2-benzimidazolethiol (2) & - \\
Curing agent & Sulfur (2) & Zinc oxide (3) \\
\end{tabular}

${ }^{*}$ Numbers in () are weight ratios in \%.

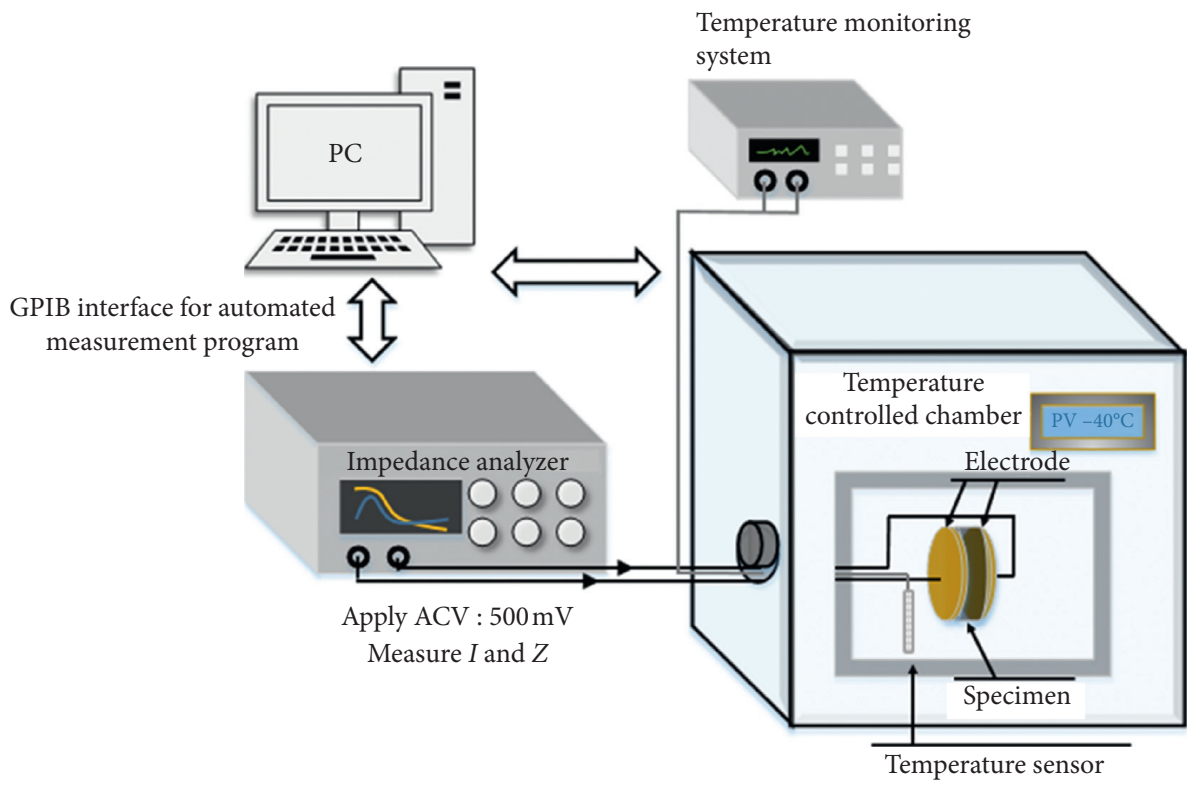

FIGURE 1: Configuration of the automatic impedance spectroscopy system for measuring the complex dielectric permittivity.

shaped specimen. $\varepsilon_{0}$ is the vacuum permittivity of $8.854 \times 10^{-12} \mathrm{~F} / \mathrm{m}$.

\subsection{Dispersion Analysis Program. A dispersion analysis} program for analyzing the complex dielectric permittivity in various polymers was developed. The program was written in C\# in the Microsoft Visual Studio Net 2018 graphical user interface (GUI). It can be executed on a Microsoft Windows system. Figure 2 shows the algorithm of the dispersion analysis program, which employs parallel Nelder-Mead optimization. The original data were composed of a set of dielectric spectra and the frequency measured at each temperature. Basically, one isothermal spectrum can be fitted with a single model function.

\subsection{Analysis Function and Relaxation Process}

2.4.1. Analysis Function. To understand the characteristics of the relaxation processes in the polymers, the experimental complex dielectric permittivity, $\varepsilon^{*}(\omega)$, can be fitted with an $\mathrm{HN}$ function. The HN function can be phenomenologically described as a combination of the conductivity term with the $\mathrm{HN}$ functional form as follows [26]:

$$
\begin{aligned}
\boldsymbol{\varepsilon}^{*}(\boldsymbol{\omega})= & \varepsilon^{\prime}-\mathbf{i} \varepsilon^{\prime \prime}=-\mathbf{i}\left(\frac{\boldsymbol{\sigma}_{\mathrm{dc}}}{\varepsilon_{0} \omega}\right)^{\mathbf{N}}+\varepsilon_{\mathrm{\infty}} \\
& +\sum_{\mathbf{k}=1,2, \ldots} \frac{\Delta \varepsilon_{\mathbf{k}}}{\left[1+\left(\mathbf{i} \omega \tau_{\mathrm{HNk}}\right)^{1-\mathbf{a}_{\mathbf{k}}}\right]^{\mathbf{b}_{\mathbf{k}}}},
\end{aligned}
$$

where the first term $\left(\sigma_{\mathrm{dc}} / \varepsilon_{0} \omega\right)^{N}$ corresponds to the conductivity, $\sigma_{\mathrm{dc}}$ is the DC conductivity, and $N$ is an exponent that characterizes the conduction process. Its contribution has the strongest influence at the highest temperature and the lowest frequency. The last term is the $\mathrm{HN}$ function. The summation symbol indicates the existence of more than one relaxation process. $\tau_{\mathrm{HNk}}$ is the average characteristic relaxation time of the corresponding process $k$. The exponents $a_{k}$ and $b_{k}$ $\left(0 \leq a_{k} \leq 1, b_{k} \leq 1\right)$ are the HN fitting shape parameters describing the distributions and shape of the relaxation times. The values of $a_{k}$ and $b_{k}$ are related to the linewidth and asymmetry, respectively, of the corresponding relaxation loss peak. As the values of $a$ and $b$ are close to 1, the linewidth and shape become broader and more symmetric, respectively. The dielectric relaxation strength, $\varepsilon_{s}-\varepsilon_{\infty}$, is represented by $\Delta \varepsilon_{k}$. 


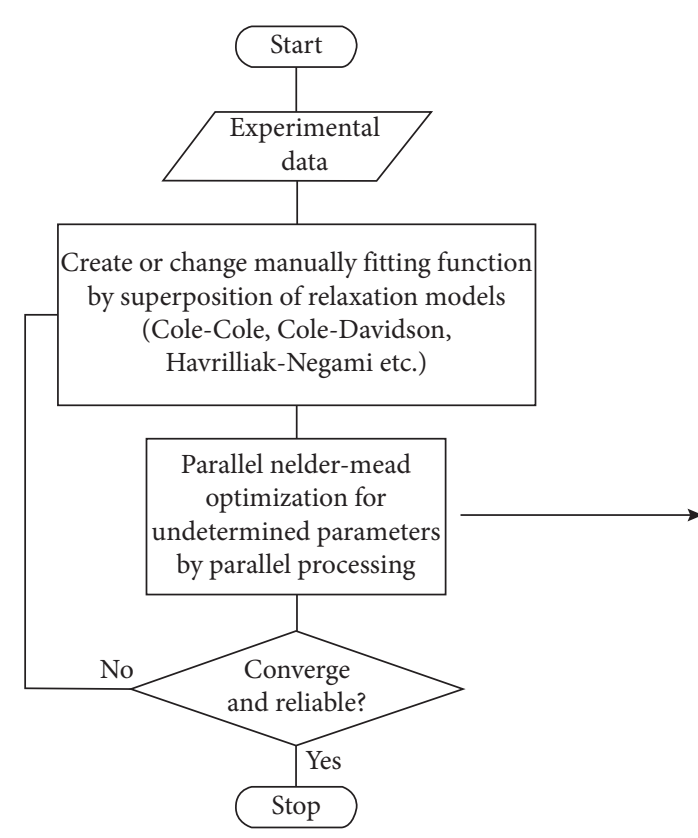

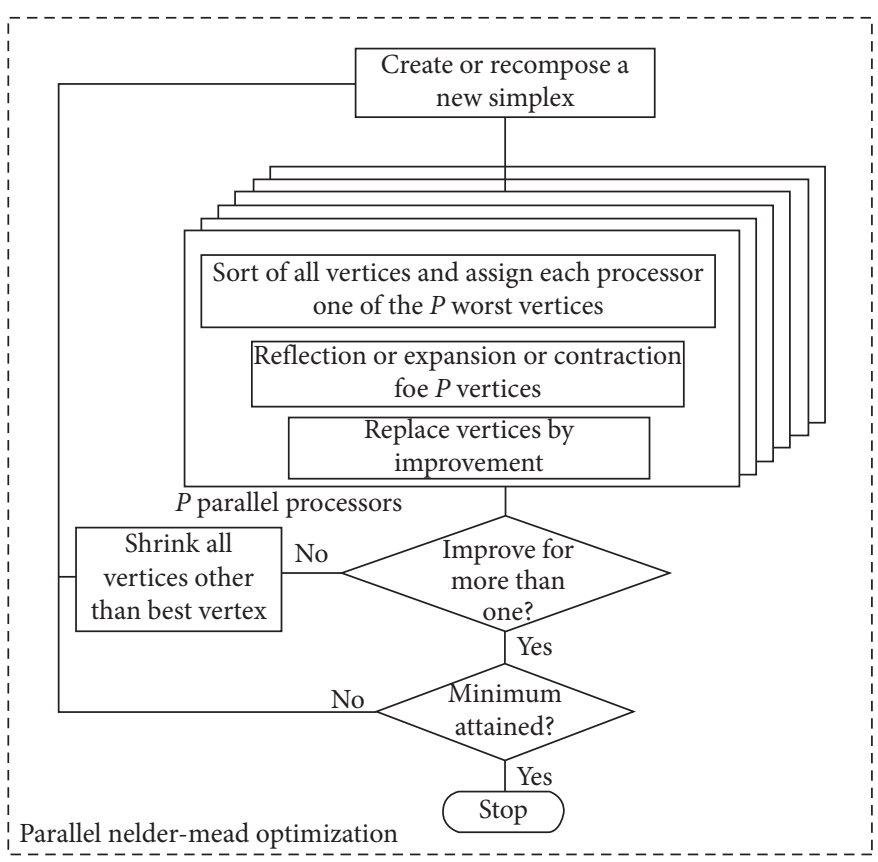

Figure 2: Algorithm of the dispersion analysis program, which employs parallel Nelder-Mead optimization.
2.4.2. Relaxation Process. The $\alpha$ relaxation responsible for the segmental motions of the main chains in the polymer is related to the glass transition [30]. The $\alpha$ relaxation reveals the following features: the asymmetric loss peak is often well described by the empirical HN function in the frequency domain, and the relaxation strength, $\Delta \varepsilon_{\alpha}$, increases with decreasing temperature. $\alpha$ relaxation is located at lower frequencies or higher temperatures than $\beta$ relaxation, indicating the stronger temperature dependency of the former. For cooperative processes, the temperature dependence of the relaxation rate obeys the Vogel-Fulcher-Tamman (VFT) law as follows [31-35]:

$$
\log f_{\alpha}=\log f_{\infty, \alpha}-\frac{A}{T-T_{0}},
$$

where $f_{\infty, \alpha}$ is the relaxation rate at an infinite frequency, $A$ is a material-specific parameter, $T$ is the temperature, and $T_{0}$ is the Vogel temperature, which is related to the dynamic glass transition process of the polymer and is usually less than $T_{\mathrm{g}}$.

$\beta$ relaxation is the secondary relaxation process in amorphous polymers [36]. $\beta$ relaxation originates from localized fluctuations of parts of the main chain or hindered rotations of polymer side groups. For most polymers, $\beta$ relaxation has been found below the glass transition temperature $\left(T_{\mathrm{g}}\right)$. The $\beta$ relaxation mechanism is a thermally activated process characterized by an Arrhenius temperature dependence of the relaxation rates $[37,38]$.

$$
f_{\beta}=f_{\infty, \beta} \exp \left[-\frac{E_{\mathrm{a}}}{k T}\right] \text {. }
$$

$E_{\mathrm{a}}$ represents the potential barrier (activation energy) between two possible equilibrium states. The activation energy $E_{\mathrm{a}}$, related to the slope of $\log f_{\beta}$ versus $1 / T$, depends on both the internal rotation barriers and the environment of a moving unit. The dielectric strength $\left(\Delta \varepsilon_{\beta}\right)$ of $\beta$ relaxation normally increases with temperature. In the frequency domain, dielectric $\beta$ relaxation displays a broad and symmetrically shaped loss peak with a halfwidth that generally narrows with increasing temperature.

The interfacial polarization effects are often called MWS effects $[39,40]$. The effects in heterogeneous systems were first highlighted by Maxwell and later modified successively by Wagner and Sillars. Interfacial polarization processes occur in heterogeneous dielectrics, especially at low frequencies, as a result of the build-up of space charges at interfaces between two media having different permittivity and conductivity values. The relaxation is also characterized by an Arrhenius temperature dependence of the relaxation rates, as shown in equation (4).

Moreover, the DC conductivity can be considered the same transport phenomena in polymers by considering the movement of different moving units (chain segment, dipole, and charge carrier). Similar to the $\alpha$ relaxation time, the temperature dependence of DC conductivity can be described by the VFT equation [41, 42]:

$$
\sigma_{\mathrm{dc}}=\sigma_{0} \exp \left[-\frac{B^{\prime}}{\mathrm{T}-\mathbf{T}_{0}^{\prime}}\right],
$$

where $B^{\prime}$ is a constant, $\sigma_{0}$ is the conductivity at a very high temperature, and $\mathbf{T}_{0}^{\prime}$ is the temperature at which the conductivity ideally reduces to zero.

The temperature dependence of DC conductivity can also be described by the Arrhenius equation [43]:

$$
\sigma_{\mathrm{dc}}=\sigma_{0} \exp \left[-\frac{E_{\mathrm{a}}}{k T}\right]
$$




\section{Results and Discussion}

3.1. NBR. Five relaxation processes, $\alpha, \alpha^{\prime}, \beta, \gamma$ and conductivity relaxations embedded in NBR, are identified for the first time from the imaginary dielectric spectra versus frequency from $0.01 \mathrm{~Hz}$ to $1 \mathrm{MHz}$ for all the temperatures investigated. Figure 3 shows an example of the decomposition of the imaginary relaxation spectra, composed of two out of five relaxation processes at $275 \mathrm{~K}$, by employing a dispersion analysis program. In Figure 3(a), a screenshot for the complex permittivity spectrum versus the frequency for the temperature range of $233 \mathrm{~K} \sim 404 \mathrm{~K}$ at $5 \mathrm{~K}$ intervals is shown. The spectrum can be decomposed by introducing two HN models with eight specific parameters, as shown in the bottom table of Figure 3(b). Several parameters involved in the optimization strategy, such as the weighting factor $\lambda$, can be adjusted in the dialog window.

The $\alpha^{\prime}, \alpha, \beta, \gamma$ and conductivity relaxations identified from the imaginary dielectric spectra for all temperatures investigated appear in the following temperature ranges.

(i) $\alpha^{\prime}$ process at temperatures ranging from $254 \mathrm{~K}$ to $404 \mathrm{~K}$

(ii) $\alpha$ process at temperatures ranging from $233 \mathrm{~K}$ to $341 \mathrm{~K}$

(iii) $\beta$ process at temperatures ranging from $233 \mathrm{~K}$ to $269 \mathrm{~K}$

(iv) $\gamma$ process at temperatures ranging from $233 \mathrm{~K}$ to $269 \mathrm{~K}$

(v) Conduction contribution at temperatures ranging from $323 \mathrm{~K}$ to $404 \mathrm{~K}$

Typical dielectric loss spectra of NBR corresponding to five relaxation processes at seven temperatures $(404 \mathrm{~K}$, $359 \mathrm{~K}, 326 \mathrm{~K}, 293 \mathrm{~K}, 272 \mathrm{~K}, 251 \mathrm{~K}$, and $233 \mathrm{~K}$ ) are presented in Figure 4, which shows the evolution of the five relaxation peaks as the temperature increases. All the relaxation peaks shift toward higher frequencies with increasing temperature. The black solid lines through the measured data (open squares) are the sums of the relaxation processes for the $\alpha^{\prime}$ (black dashed line), $\alpha$ (red dashed line), $\beta$ (blue dashed line), and $\gamma$ (gray dashed line) processes fitted by the HN permittivity functions and conductivity (navy dashed line) process by the fitted conductivity function of the first term in equation (2). As an example, the loss spectra at $404 \mathrm{~K}$ are composed of conductivity and $\alpha^{\prime}$ processes. The loss spectra at other temperatures are also composed of two or three relaxation processes.

Figure 4 depicts the evolution of typical $\alpha^{\prime}$ dielectric spectra at five representative temperatures, excluding $251 \mathrm{~K}$ and $233 \mathrm{~K}$, and it displays primary $\alpha$ dielectric spectra detected in a temperature range from $233 \mathrm{~K}$ to $341 \mathrm{~K}$ at five representative temperatures $(326 \mathrm{~K}, 293 \mathrm{~K}, 272 \mathrm{~K}, 251 \mathrm{~K}$, and $233 \mathrm{~K})$. The $\alpha$ and $\alpha^{\prime}$ processes at these temperatures are well fitted by the $\mathrm{HN}$ equation. The $\alpha$ relaxation process induced by the small dipole moment is responsible for the segmental motions of the main chains from the butadiene and acrylonitrile structures caused by the rotation of the $\mathrm{C}-\mathrm{C}$ bonds under the applied electric field. The $\alpha^{\prime}$ process is usually called normal-mode relaxation, which was observed in a Stockmayer type A polymer with a dipole moment vector parallel to the principal axis. The $\alpha^{\prime}$ process originates from the entire fluctuation of the end-to-end vector. The proposed $\alpha$ and $\alpha^{\prime}$ relaxation processes in the NBR molecular chain structure are shown in Figure 5.

Additional relaxations called secondary relaxations, that is, $\beta$ and $\gamma$ processes with faster time scales, are observed at low temperatures from $233 \mathrm{~K}$ to $269 \mathrm{~K}$. The $\beta$ relaxation versus frequency displays a loss peak with a symmetrical shape, which is ascribed to the motion of the cyanide side group with a larger dipole moment. Moreover, conduction spectra are detected. Figure 4 depicts the imaginary dielectric spectra containing the $\alpha$ and $\alpha^{\prime}$ processes at three representative temperatures $(404 \mathrm{~K}, 359 \mathrm{~K}$, and $326 \mathrm{~K})$. At these temperatures, the navy dashed lines are fitted by the conductivity contribution of the first term of equation (2). At high temperatures above $323 \mathrm{~K}$ and low frequencies less than a few hundred $\mathrm{kHz}$, a sharp increase in the imaginary dielectric spectra with decreasing frequency is correlated with the conduction of charges, which mainly occurs in polymers due to highly conductive filler.

According to the fitting of equation (2), the relaxation strength $\left(\Delta \varepsilon_{k}\right)$ and shape parameters $(a$ and $b)$ for the four relaxation processes except conductivity are plotted as a function of temperature, as shown in Figure 6. In the $\alpha^{\prime}$ process (Figure 6(a)), $\Delta \varepsilon_{\alpha^{\prime}}$ decreases with increasing temperature. This temperature dependence is predicted by the Debye theory of dielectric relaxation, as improved by Bottcher et al. [44, 45]. The value of $a$ lies in the range of $0.2 \sim 0.5$, and the value of $b$ is $\sim 1.0$ (Figure $6(\mathrm{~b})$ ). This finding implies that the linewidth is relatively sharp with a linewidth of $10^{5} \mathrm{~Hz} 10^{6} \mathrm{~Hz}$, and its shapes are symmetrical.

For the $\alpha$ process (Figures 6(c) and 6(d)), $\Delta \varepsilon_{\alpha}$ decreases with increasing temperature. Similar to $\alpha^{\prime}$, this temperature dependence is also predicted by the Debye theory of dielectric relaxation. $a$ and $b$ are determined to be $0.0 \sim 0.9$ and $0.5 \sim 1.0$, respectively. This finding implies again that the linewidth is more or less broad $\left(10^{4} \mathrm{~Hz} \sim 10^{15} \mathrm{~Hz}\right)$ and the shape is symmetrical. $\Delta \varepsilon_{\beta}$ and $\Delta \varepsilon_{\gamma}$ for secondary relaxation processes $(\beta$ and $\gamma)$, as shown in Figures 6(e) and $6(\mathrm{~g})$, are smaller than those for the primary relaxation process, as expected. The $a$ values (Figures 6(f) and 6(h)) for the $\beta$ and $\gamma$ processes are 0.25 and $\sim 0.5$, respectively, corresponding to lines of $10^{6} \mathrm{~Hz}$ and $\sim 10^{15} \mathrm{~Hz}$. The $b$ value for the $\beta$ process is found to be 1, as shown in Figure 6(f), indicating that the process complies with the Cole-Cole function rather than the $\mathrm{HN}$ function. The $b$ value for the $\gamma$ process (Figure $6(\mathrm{~h})$ ) is found to be less than 0.5 , and the relaxation loss spectra are asymmetrical.

In the case of conductivity relaxation, the $\mathrm{DC}$ conductivity $\sigma_{\mathrm{dc}}$ and exponent $N$ are determined as a function of temperature as a result of fitting the first term of equation (2). The determined $N$ value for the temperature variations is nearly 1 , implying that $\sigma_{\mathrm{dc}}$ is a constant independent of the frequency.

The central frequency $\left(f_{0}\right)$ of the imaginary loss peak for the corresponding four relaxation processes, $\alpha^{\prime}, \alpha, \beta$, and $\gamma$, and the conduction contribution are plotted as a function of 


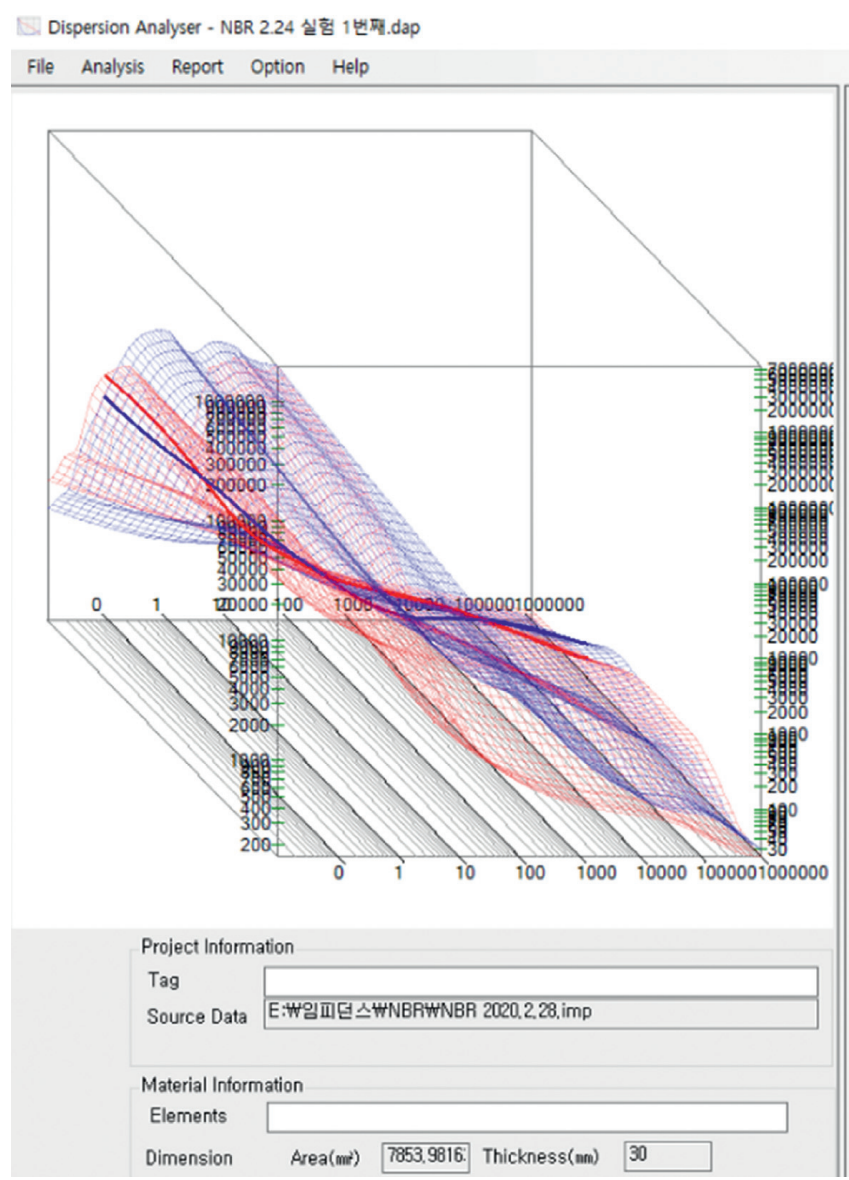

(a)

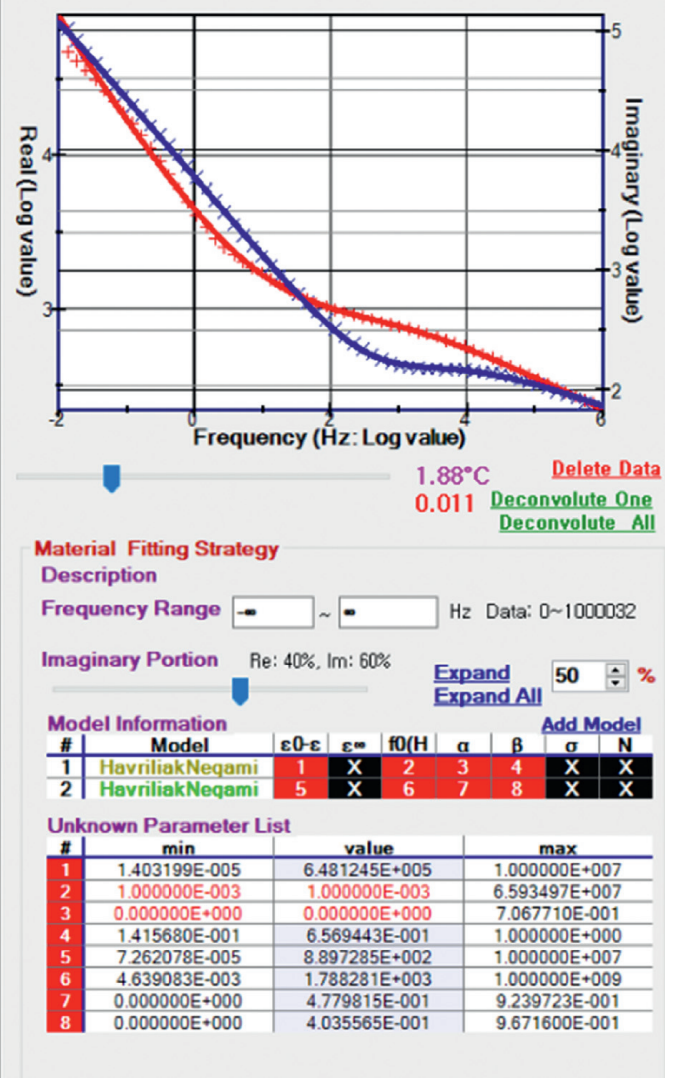

(b)

Figure 3: An example of the decomposition of two relaxation processes in NBR by the dispersion analysis program at $275 \mathrm{~K}$. (a) Real (red line) and imaginary (blue line) fitted permittivity values versus the frequency for all temperatures and (b) the decomposition of the two relaxation processes by the dispersion analysis program. In (b), the measured real (red $\times$ ) and imaginary (blue $\times$ ) permittivity and the fitted real (red line) and imaginary (blue line) permittivity graphs at $275 \mathrm{~K}$ are shown, and their fitted parameters are listed at the bottom table.

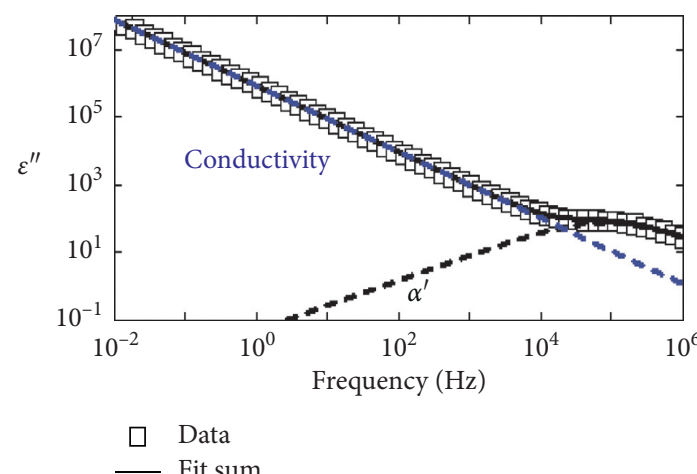

(a)

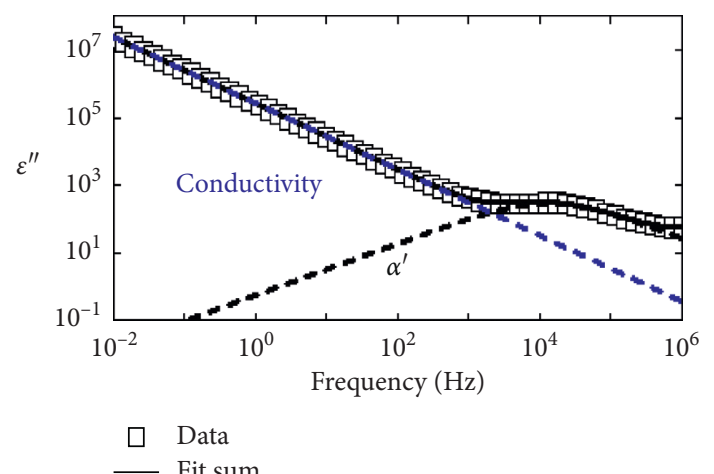

(b)

Figure 4: Continued. 


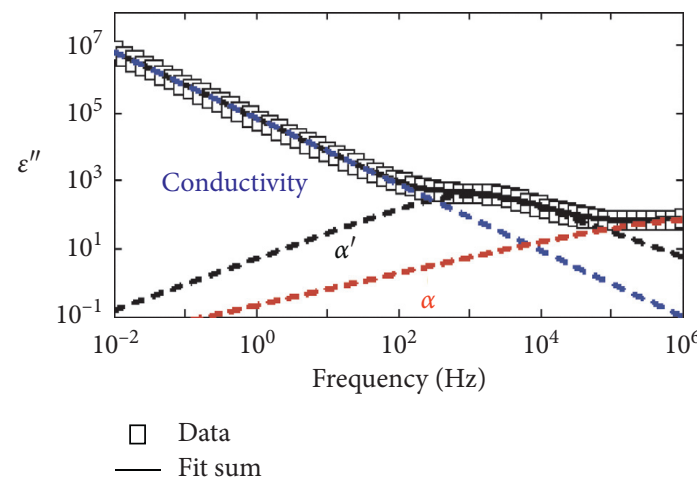

(c)

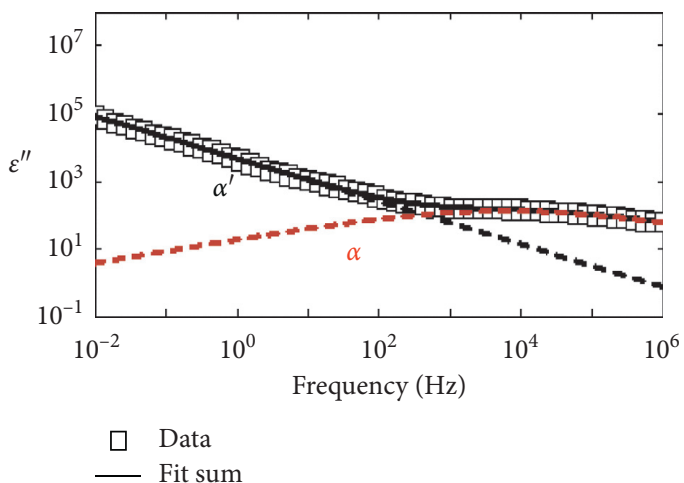

(e)

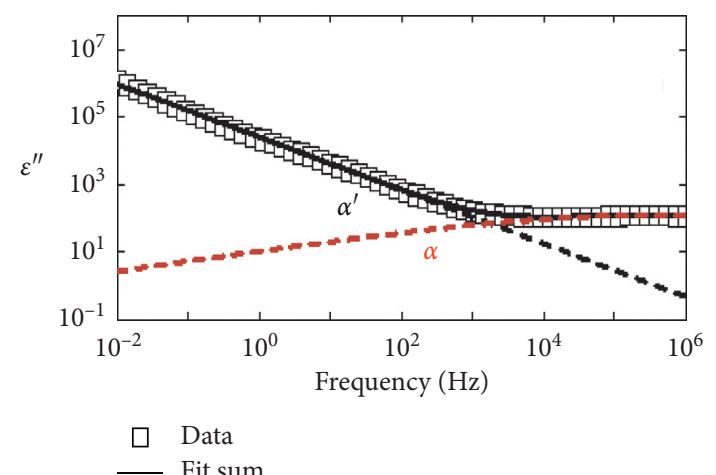

(d)

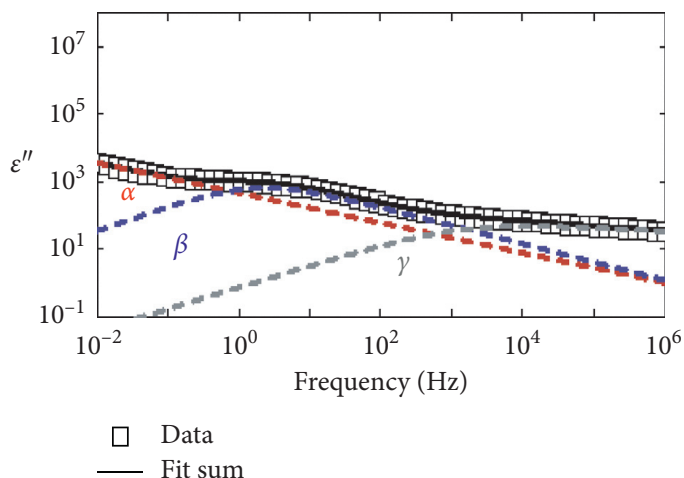

(f)

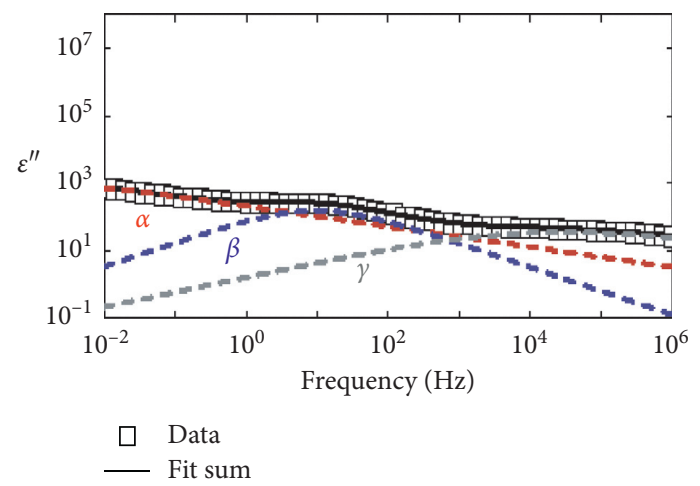

(g)

Figure 4: Decomposed relaxation process of NBR for seven representative temperatures (a) $404 \mathrm{~K}$ (b) $359 \mathrm{~K}$ (c) $326 \mathrm{~K}$ (d) $293 \mathrm{~K}$ (e) $272 \mathrm{~K}$ (f) $251 \mathrm{~K}$, and (g) $233 \mathrm{~K}$. Five relaxation processes, $\alpha^{\prime}$ (black dashed line), $\alpha$ (red dashed line), $\beta$ (blue dashed line), $\gamma$ (gray dashed line), and conduction (navy dashed line), contribute to the imaginary permittivity data.

the reciprocal temperature, as shown in Figure 7, which is the relaxation map. The $f_{0}$ value for the four relaxation processes decreases with decreasing temperature, as also shown in Figure 4. Simultaneously, the fitting results (solid lines) obtained by assuming the Arrhenius temperature dependence in equation (4) for the $\alpha^{\prime}$ relaxations give activation energies $E_{\mathrm{a}, \alpha^{\prime}}=69.6 \mathrm{~kJ} / \mathrm{mol}$. The activation energy for the $\alpha^{\prime}$ process is comparable to the $E_{\mathrm{a}}=83.1 \mathrm{~kJ} / \mathrm{mol}$ value obtained from the thermogravimetric analysis [46]. Similar to the $\alpha^{\prime}$ process, the activation energies for the $\beta$ and $\gamma$ processes determined by assuming the Arrhenius temperature dependence are $E_{\mathrm{a}, \beta}=32.0 \mathrm{~kJ} / \mathrm{mol}$ and $E_{\mathrm{a}, \gamma}=24.3 \mathrm{~kJ} /$ mol, respectively, which is the typical value of the activation energy.

On the other hand, the relaxation rate of the $\alpha$ relaxation process is normally fitted by the VFT law, as shown in equation (3), giving rise to the glass temperature, $T_{\mathrm{g}}=242.4 \mathrm{~K}$, as shown in Figure 7 . The obtained glass transition temperature $T_{\mathrm{g}}$ is very similar to the $242.2 \mathrm{~K}$ measurement of the differential scanning calorimetry.

As a result of fitting the first term of equation (2) for the conductivity contribution, the DC conductivity $\sigma_{\mathrm{dc}}$ is determined as a function of temperature; as shown in Figure 7 $\sigma_{\mathrm{dc}}$ is found to increase with increasing temperature, which 


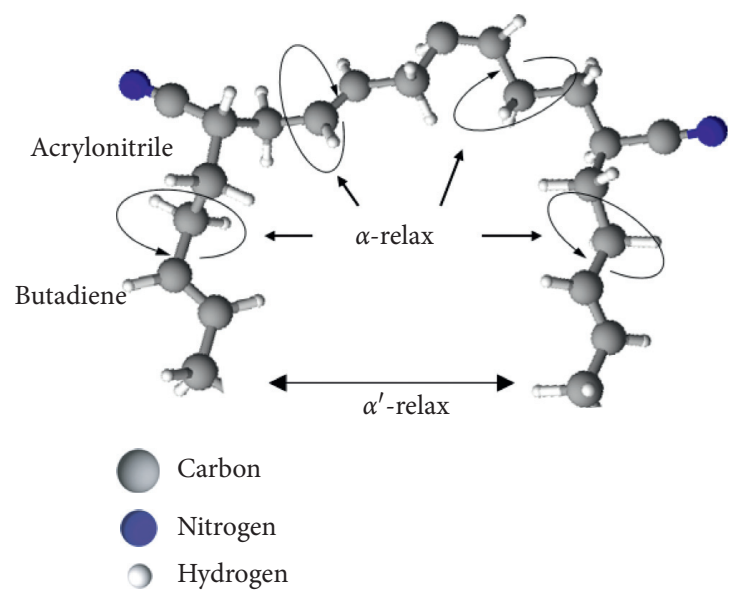

FIgURE 5: Proposed $\alpha$ and $\alpha^{\prime}$ relaxation processes in the NBR molecular structure.

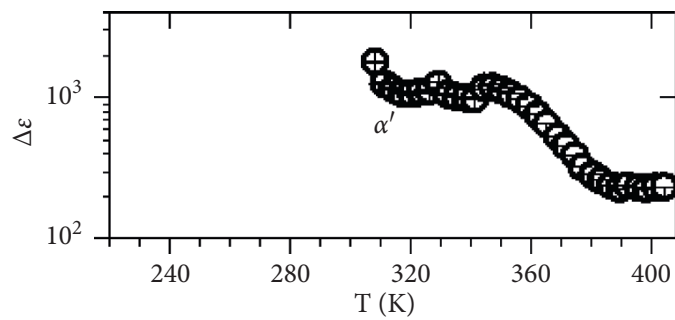

(a)

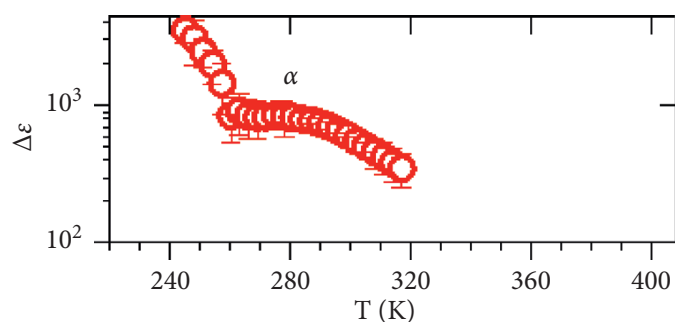

(c)

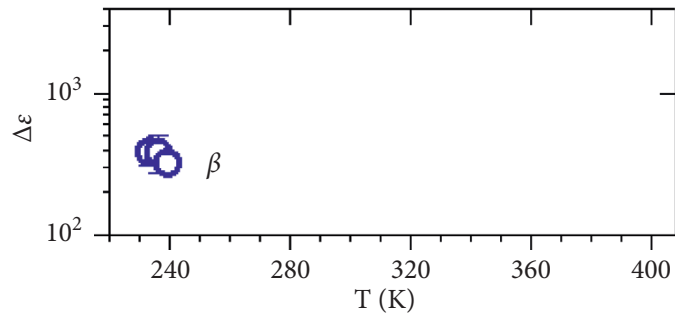

(e)

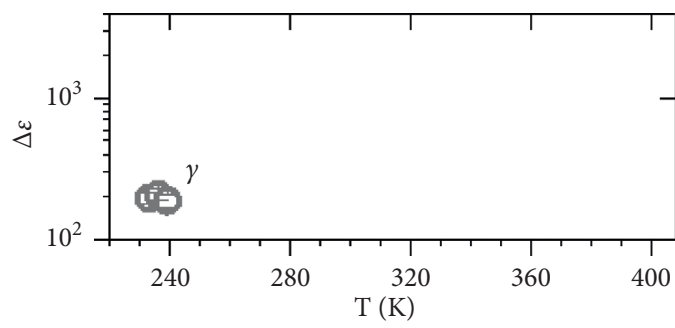

(g)

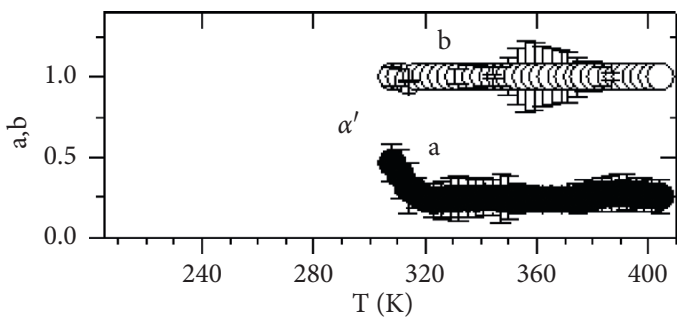

(b)

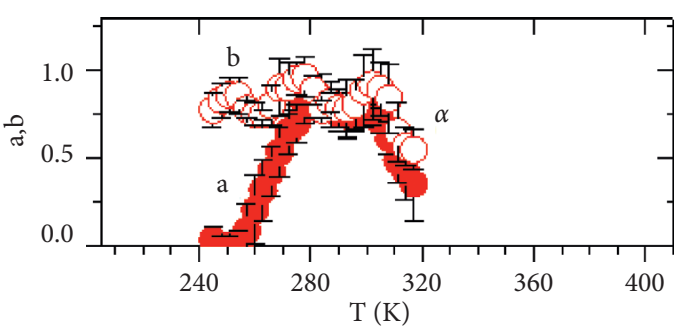

(d)

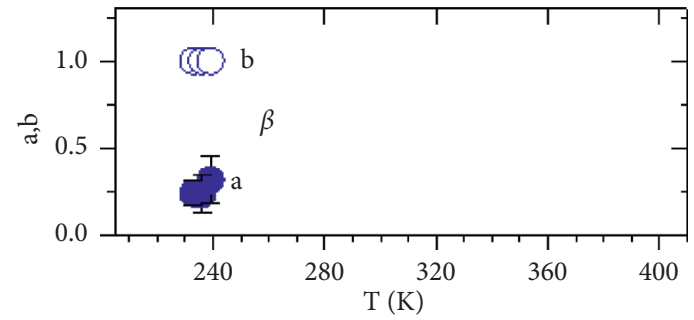

(f)

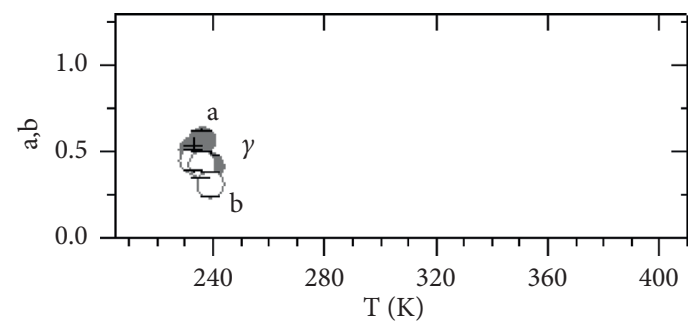

(h)

FIGURE 6: Temperature dependence of the relaxation strength and shape parameter for the four relaxation processes in NBR. 


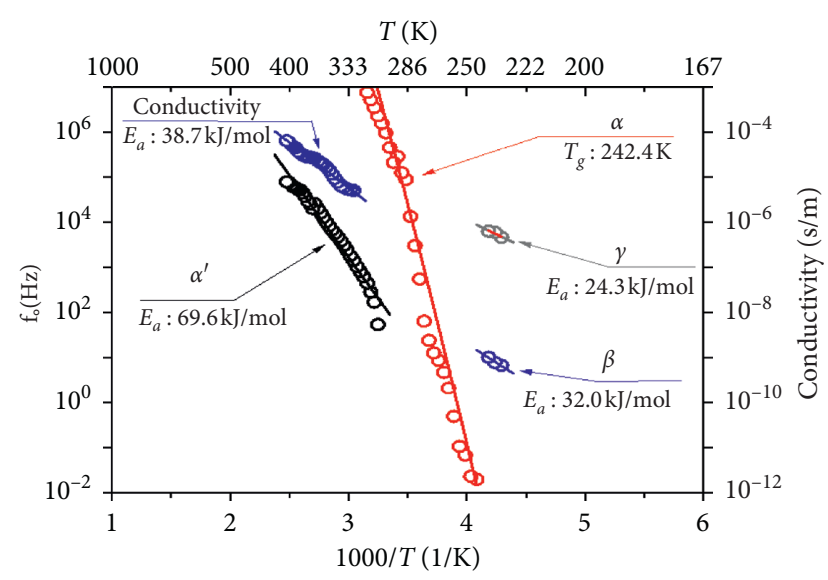

FIGURE 7: Reciprocal temperature dependence of the central frequency $\left(f_{0}\right)$ of the imaginary loss peak for the $\alpha^{\prime}$ (black circle), $\alpha$ (red circle), $\beta$ (blue circle), $\gamma$ (gray circle), and conductivity (navy circle) processes in NBR. The corresponding solid lines are the fitted results for the relaxation processes.

confirms the Arrhenius dependence as expected. The reciprocal temperature dependence of conductivity satisfies the Arrhenius temperature dependence of equation (6), which gives an activation energy of $E_{\mathrm{a}, \mathrm{c}}=38.7 \mathrm{~kJ} / \mathrm{mol}$.

3.2. EPDM. The complex dielectric permittivity for the EPDM specimen is measured in the $0.01 \mathrm{~Hz}$ to $1 \mathrm{MHz}$ and $233 \mathrm{~K}$ to $404 \mathrm{~K}$ ranges. Similar to NBR, we also decompose the superimposed dielectric spectra by employing the dispersion analysis program. In Figure 8(a), a screenshot for the complex permittivity spectrum for all temperatures with a $5 \mathrm{~K}$ interval is shown. Figure $8(\mathrm{~b})$ shows an example of the decomposition of relaxation spectra at $272 \mathrm{~K}$, which comprises three relaxation processes. The decomposition procedure is performed by the three $\mathrm{HN}$ functions, as shown in Figure 8(b).

The five relaxation processes, $\beta, \alpha, \alpha \beta$, MWS, and conductivity, are determined from imaginary dielectric spectra for all the temperatures investigated. The relaxation processes are observed following the temperature ranges.

(i) $\alpha$ relaxation process at temperatures ranging from $233 \mathrm{~K}$ to $302 \mathrm{~K}$

(ii) $\beta$ relaxation process at temperatures ranging from $233 \mathrm{~K}$ to $305 \mathrm{~K}$

(iii) The $\alpha \beta$ merged relaxation at temperatures ranging from $305 \mathrm{~K}$ to $404 \mathrm{~K}$ originating from the $\beta$ process

(iv) MWS relaxation process at temperatures ranging from $233 \mathrm{~K}$ to $404 \mathrm{~K}$

(v) Conduction contribution at temperatures ranging from $308 \mathrm{~K}$ to $404 \mathrm{~K}$.

Typical dielectric loss spectra of EPDM at seven temperatures $(404 \mathrm{~K}, 374 \mathrm{~K}, 344 \mathrm{~K}, 313 \mathrm{~K}, 281 \mathrm{~K}, 257 \mathrm{~K}$, and $233 \mathrm{~K}$ ) are displayed in Figure 9, which shows the evolution of four relaxation peaks as the temperature increases. The black solid lines through the data (open squares) are the fitted sums of the MWS (orange dashed line), $\beta$ (blue dashed line) processes by two $\mathrm{HN}$ fits, and the conductivity process (navy dashed line) by the fitted conductivity function (404 K, $374 \mathrm{~K}, 344 \mathrm{~K}$, and $313 \mathrm{~K}$ ) or the fitted sums of the MWS (orange dashed line), $\alpha$ (red dashed line), and $\beta$ (blue dashed line) processes by three $\mathrm{HN}$ fits $(281 \mathrm{~K}, 257 \mathrm{~K}$, and $233 \mathrm{~K})$. The $\alpha \beta$ merged relaxation process from $305 \mathrm{~K}$ to $404 \mathrm{~K}$ originates from the $\beta$ process. All the relaxation peaks shift toward a higher frequency with increasing temperature.

The $\alpha$ dielectric spectra of the EPDM specimen are observed below room temperature. Each dielectric loss spectrum versus frequency displays a wide loss peak with a symmetrical shape, which is attributed to the relaxation of local segmental motion of the main chain. The $\beta$ relaxation spectra detected in the entire temperature range investigated display a narrower loss peak than that of the $\alpha$ process with a symmetrical shape. The $\beta$ process is attributed to the relaxation of the rotational motion of the side groups. The $\alpha$ process is responsible for the rotation of the main chain $(\mathrm{C}-$ $\mathrm{C}$ bond) in the ethylene, propylene, and ENB units, as shown in Figure 10. The $\beta$ relaxation process is based on the reorientation of the $\mathrm{CH}_{3}$ bond (methyl) in the side chain of the propylene structure.

The $\alpha$ relaxation spectra are well fitted by the Cole-Cole function with $b=1$ in equation (2). Figure 11(a) shows the temperature dependence of $a$ for the $\alpha$ process obtained from the dispersion analysis program. The $a$ values for the $\alpha$ process are independent of temperature. The value for $a$ is found to be 0.85 with $b=1$. This finding implies that relaxation spectra have a symmetrical shape with a linewidth above $10^{15} \mathrm{~Hz}$.

The $\beta$ relaxation spectra detected in the entire temperature range investigated display a narrower loss peak compared to the $\alpha$ process with a symmetrical shape, fitted by the Cole-Cole equation in the case of $b=1$ in equation (2). Figure 11(b) shows the temperature dependence of the shape parameter obtained from the dispersion simulation with equation (2). The $a$ value for the $\beta$ process remains constant for temperature variations within the measurement uncertainty. The values for $a$ are found to be $0.50 \sim 0.65$, corresponding to a linewidth of $10^{12} \mathrm{~Hz} \sim 10^{14} \mathrm{~Hz}$ with a symmetrical shape.

As a result of a simulation from the dispersion analysis program, the $\Delta \varepsilon$ values for both the $\alpha$ and $\beta$ processes as a function of temperature are also determined, as shown in Figure 11(c). The relaxation strength shows $\Delta \varepsilon_{\alpha}>\Delta \varepsilon_{\beta}$ for all the temperatures investigated. This is generally observed for most amorphous polymers having a dipole moment attached to the main chain. For the $\alpha$ process, $\Delta \varepsilon_{\alpha}$ decreases with increasing temperature. This temperature dependence is predicted by the Debye theory of dielectric relaxation. However, the $\Delta \varepsilon_{\beta}$ value for the $\beta$ relaxation process increases with temperature, which is caused by an increase in the fluctuation angle of the dipole vector or by the number of moving dipoles increasing the temperature. These are also general behaviors for the $\beta$ process. The peaks of the $\alpha$ and $\beta$ processes, which are separated below $305 \mathrm{~K}$ (indicated by arrows), merge above this temperature, as shown in Figure 11(c). The merged $\alpha$ and $\beta$ processes are called the $\alpha \beta$ 


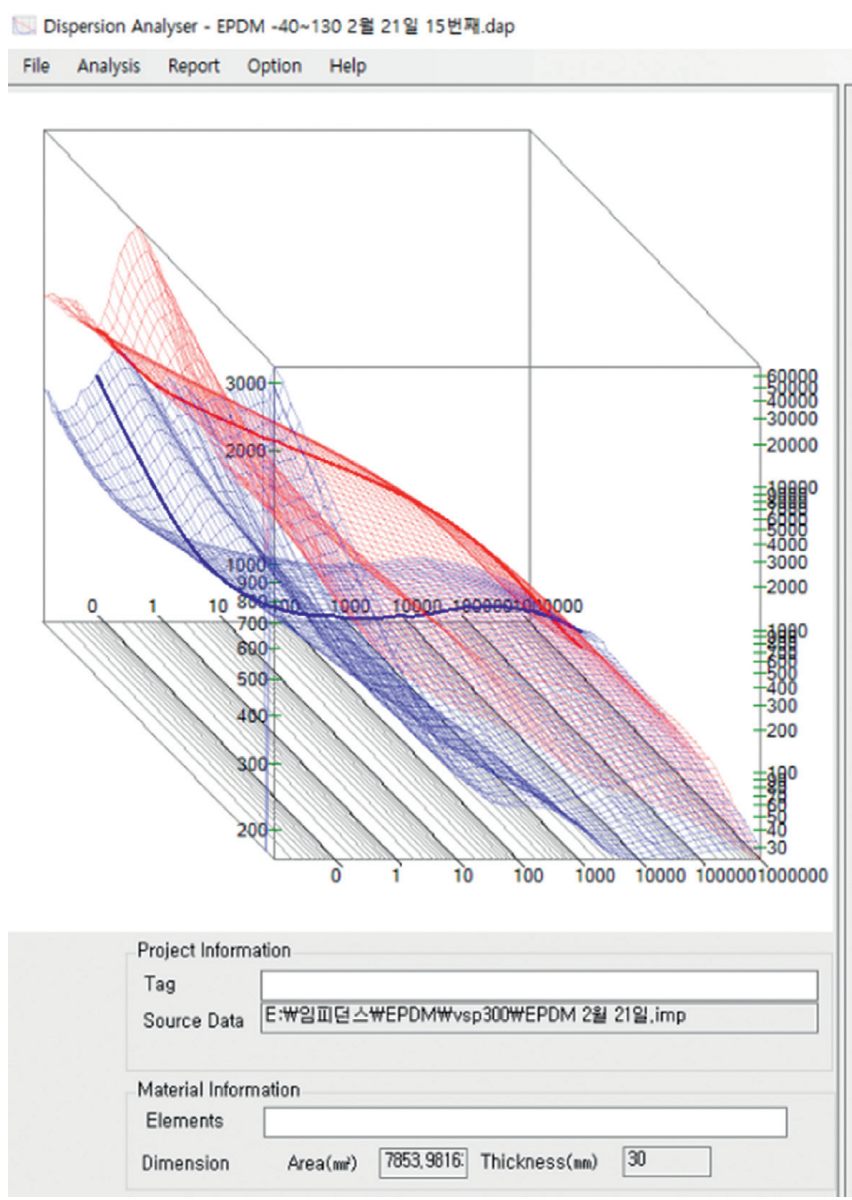

(a)

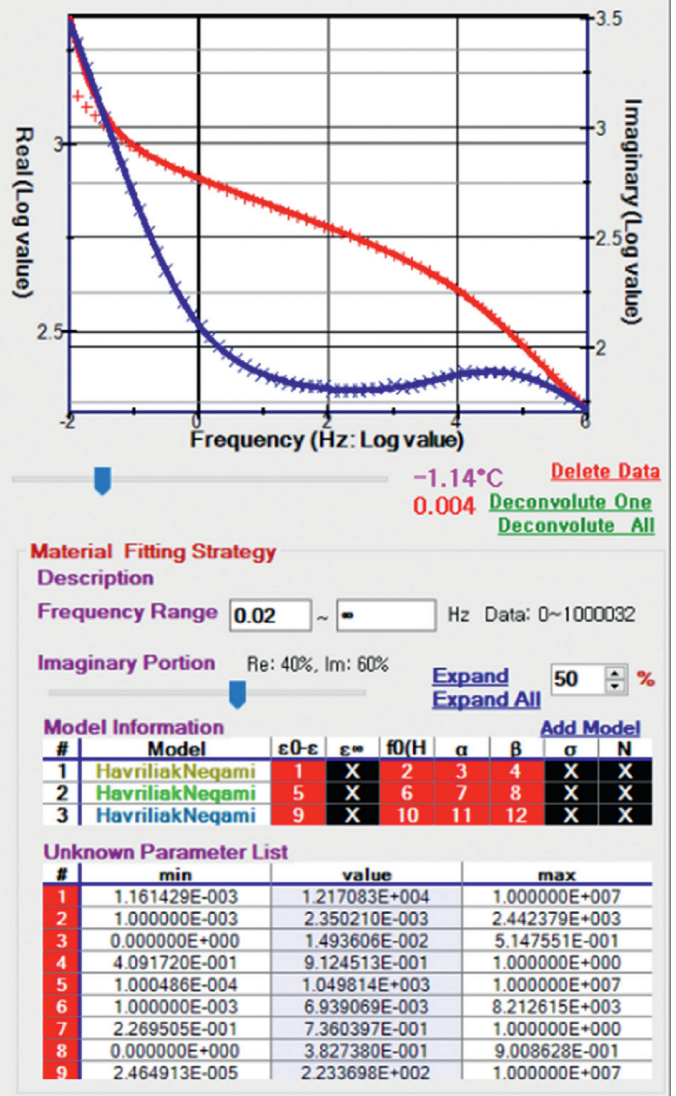

(b)

FIGURE 8: An example of the decomposition of relaxation spectra by the dispersion analysis program at $272 \mathrm{~K}$ in EPDM. (a) Real (red line) and imaginary (blue line) fitted permittivity line versus frequency for all temperatures and (b) the decomposition of three relaxation processes by the dispersion analysis program. In (b), the measured data of the real (red $\times$ ) and imaginary (blue $\times$ ) permittivity and the fitted real (red line) and imaginary (blue line) permittivity graphs at $272 \mathrm{~K}$ are shown, and their fitted parameters are listed in the bottom table.

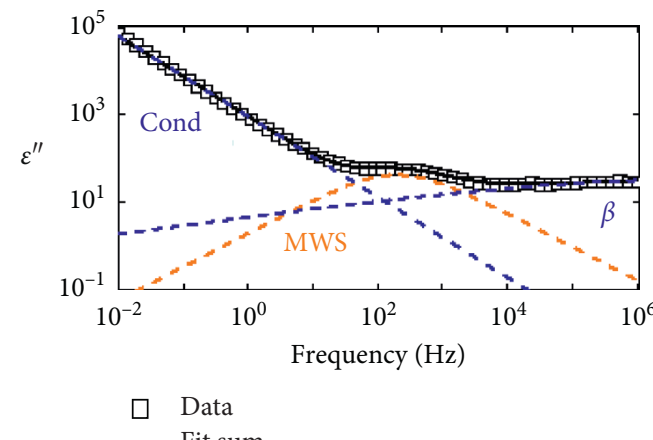

(a)

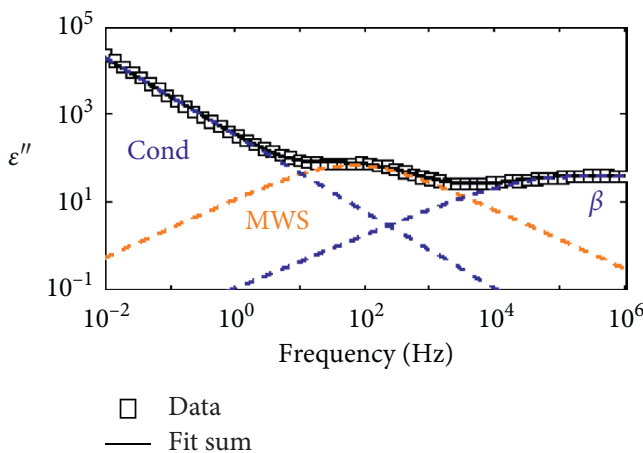

(b)

Figure 9: Continued. 


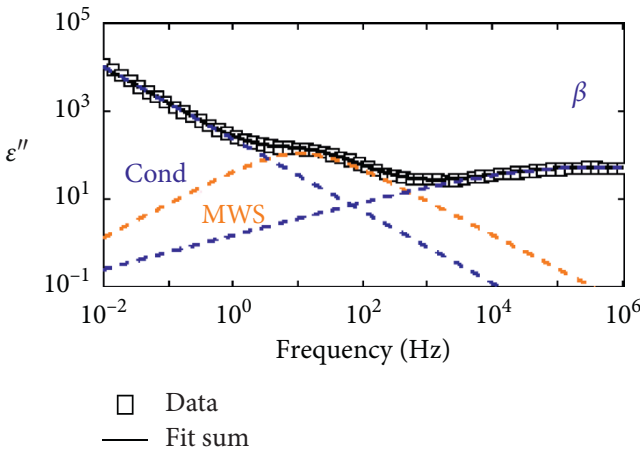

(c)

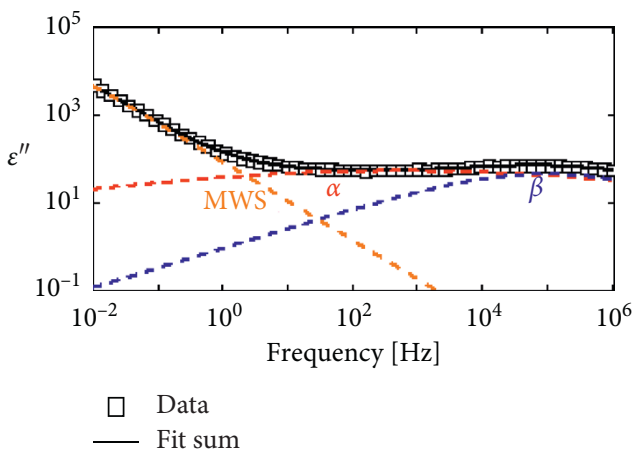

(e)

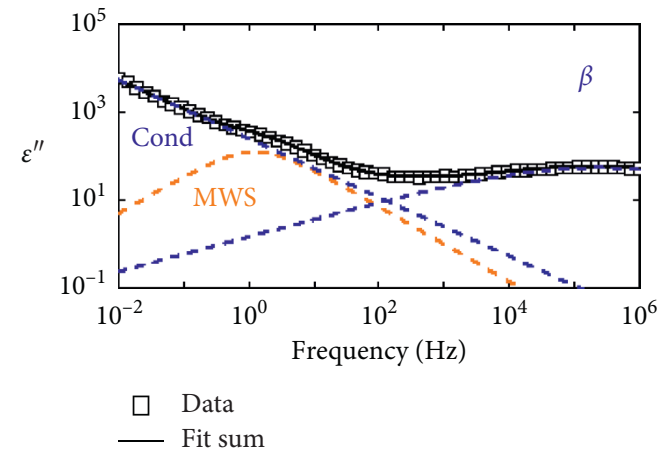

(d)

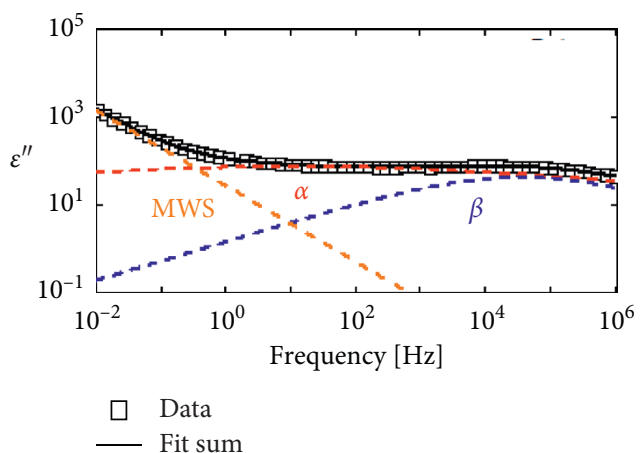

(f)

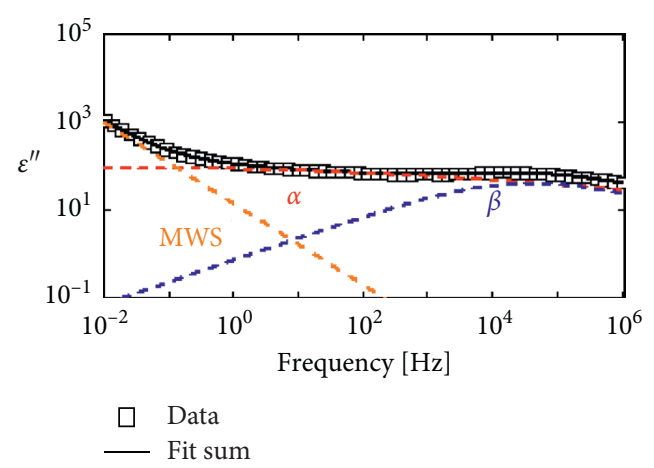

(g)

FigURE 9: Relaxation processes of the EPDM specimen at seven temperatures ranging from $233 \mathrm{~K}$ to $404 \mathrm{~K}$. Four relaxation processes, $\alpha$ (red dashed line), $\beta$ (blue dashed line), MWS (orange dashed line), and conductivity (navy dashed line), contribute to the imaginary permittivity data. (a) $404 \mathrm{~K}$ (b) $374 \mathrm{~K}$ (c) $344 \mathrm{~K}$ (d) $313 \mathrm{~K}$ (e) $281 \mathrm{~K}$ (f) $257 \mathrm{~K}$, and (g) $233 \mathrm{~K}$.

process. However, Figure 11(c) shows that the temperature behavior for the merged peak may follow that for the $\beta$ process.

MWS spectra are detected in the entire temperature range investigated. Each dielectric loss spectrum versus frequency displays a clear loss peak with a symmetrical shape above $290 \mathrm{~K}$ (Figure 9), which may be attributed to the interfacial interaction between the filler and polymer matrix. This MWS relaxation may be due to the accumulation of charged ions at the interfaces of inhomogeneous media. This relaxation arises from the free ingredients added to EPDM, which are present at the stage of processing and are now immobilized in the specimen, which leads to conductivity, and consequently, the charges can migrate under the applied electric field. MWS relaxation is usually confounded with ionic conduction, as these two phenomena originate from charged carrier mobility.

The MWS relaxation spectra are fitted with the $\mathrm{HN}$ function in equation (2). Figure 12(a) shows the temperature dependence of $a$ and $b$ obtained from the dispersion analysis program. The values for $a$ for the MWS relaxation spectra range from $0 \sim 0.4$. The relaxation spectra have narrow linewidths in the range from $10^{4} \mathrm{~Hz} \sim 10^{6} \mathrm{~Hz}$. The linewidth increases with increasing temperature. The shape parameter $b$ is found to be 1 at all the temperatures investigated, implying that the imaginary permittivity for MWS relaxation is symmetrical with respect to the frequency. This finding again indicates that this behavior of the relaxation 


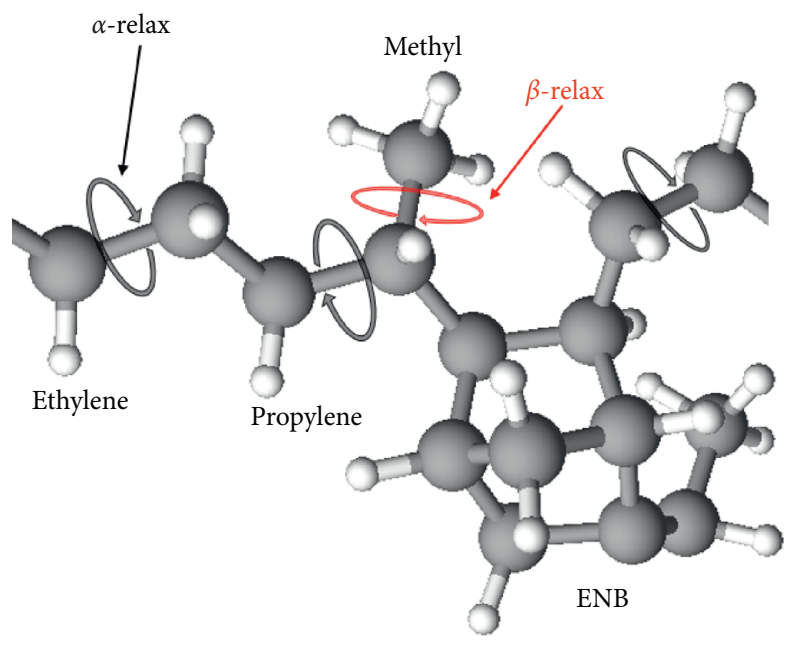

Carbon
Hydrogen

Figure 10: Proposed $\alpha$ and $\beta$ relaxation processes in the EPDM molecular structure.

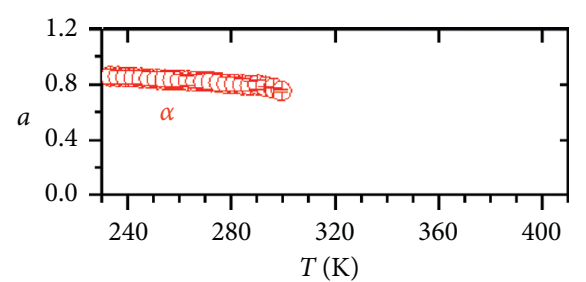

(a)

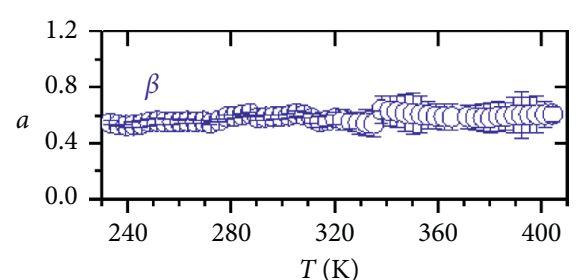

(b)

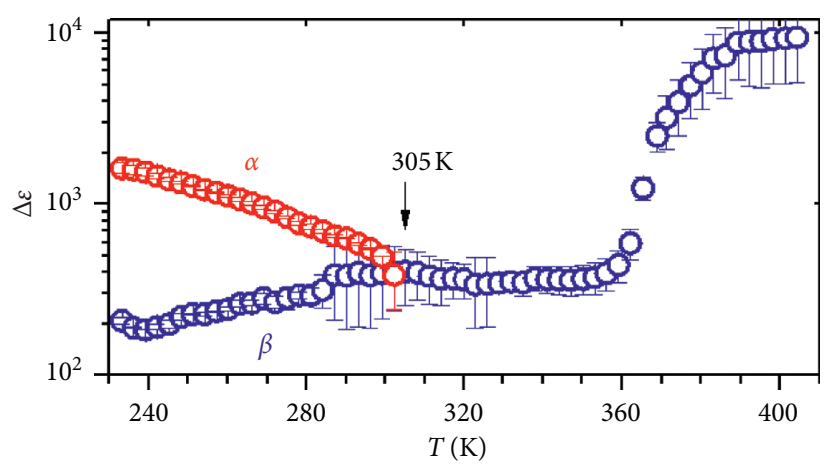

(c)

FIgURE 11: Temperature dependence of the (a), (b) shape parameter and (c) relaxation strength for the $\alpha$ and $\beta$ processes.

spectra also satisfies the Cole-Cole equation. Moreover, Figure 12(b) shows that $\Delta \varepsilon_{\mathrm{MWS}}$ for the MWS relaxation process decreases with temperature, similar to that of the $\alpha$ process.

Conductivity spectra are detected above room temperature, which is shown in Figure 9 at the four representative temperatures ( $404 \mathrm{~K}, 374 \mathrm{~K}, 344 \mathrm{~K}$, and $313 \mathrm{~K}$ ). The conductivity spectra are attributed to the conductive carbon black filler contained in EPDM. It is normally observed that the imaginary permittivity increases with decreasing frequency and with increasing temperature. Two relaxation processes corresponding to both the MWS polarization and conductivity are normally observed in the composite containing conducting filler. The conductivity (navy solid lines) through the data (open square) is the fitting result by the first term of equation (2), and the DC conductivity $\sigma_{\mathrm{dc}}$ and exponent $N$ are determined as a function of temperature. The determined $N$ value is nearly $0.85 \sim 0.95$, implying that $\sigma_{\mathrm{dc}}$ is almost constant and independent of the frequency. 


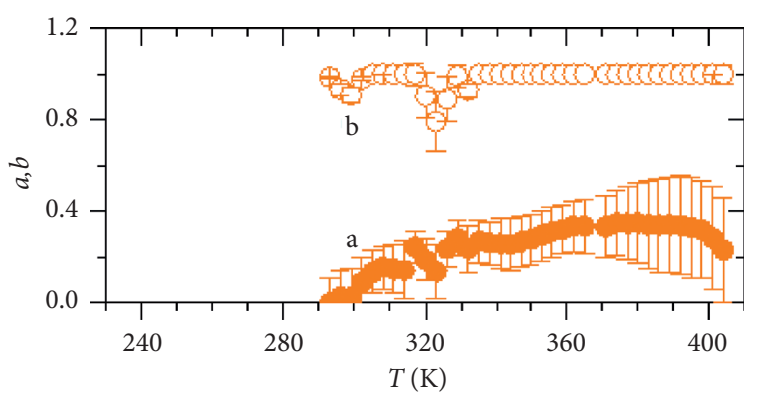

(a)

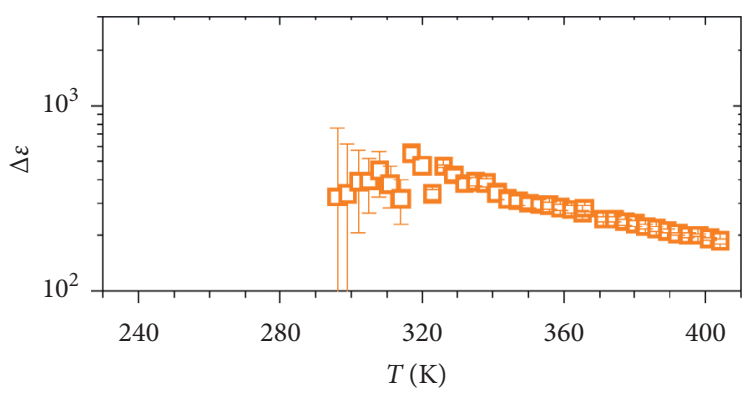

(b)

Figure 12: Temperature dependence of the (a) shape parameter and (b) relaxation strength for the MWS relaxation spectra.

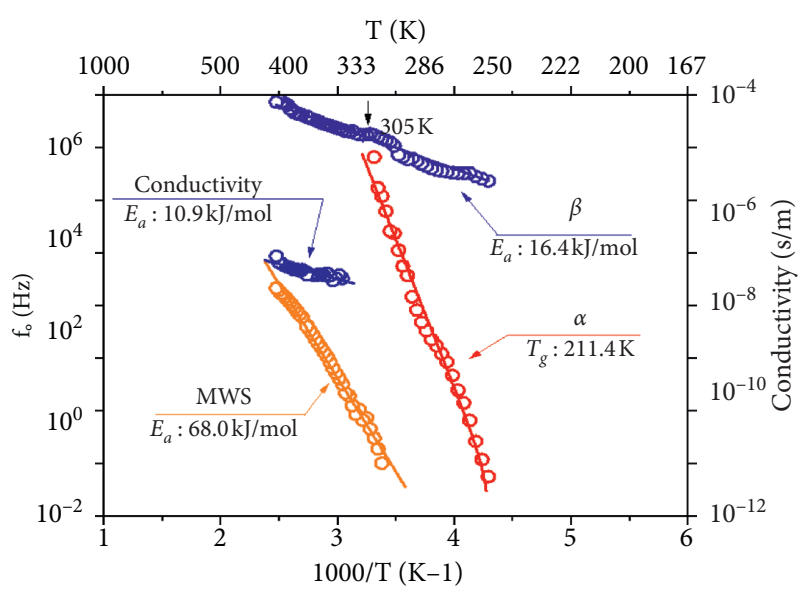

FIGURE 13: Reciprocal temperature dependence of the central frequency $\left(f_{0}\right)$ of the imaginary loss peak for $\alpha$ (red circles), $\beta$ (blue circles), MSW (orange circles), and conductivity (navy circles) processes in EPDM. The corresponding solid lines are the fitted results for the relaxation processes.

The $f_{0}$ values of the imaginary loss peak for the corresponding $\alpha, \beta$ and MWS relaxation processes are plotted as a function of the reciprocal temperature, as shown in Figure 13. The fitting results (solid line) of these relaxations are also displayed. According to equation (4), the Arrhenius temperature dependence of $f_{0}$ in the relaxation rates for the $\beta$ and MWS relaxation processes results in $E_{\mathrm{a}, \beta}=16.4 \mathrm{~kJ} / \mathrm{mol}$ and $E_{\mathrm{a}, \mathrm{MWs}}=68.0 \mathrm{~kJ} / \mathrm{mol}$, respectively. By assuming a nonArrhenius temperature dependence of the $\alpha$ relaxation rate, the relaxation rate can be fitted by the VFT law, as shown in equation (3); this approach provides glass transition temperature values of $T_{\mathrm{g}}=211.4 \mathrm{~K}$ (Figure 13).

The navy solid lines through the data (navy open squares) are the fitting results by the first term of equation (2), and the DC conductivity $\sigma_{\mathrm{dc}}$ is determined as a function of the temperature, as shown in Figure $13 \sigma_{\mathrm{dc}}$ is found to increase with increasing temperature, which follows the Arrhenius dependence as expected. The reciprocal temperature dependence of the conductivity data fit by equation (6) gives activation energy of $E_{\mathrm{a}}=10.9 \mathrm{~kJ} / \mathrm{mol}$.

The $\alpha \beta$ peaks that merge in the temperature range from $305 \mathrm{~K}$ to $404 \mathrm{~K}$ are separated below approximately $305 \mathrm{~K}$ (Figure $11(\mathrm{c})$ ). The intersection at $305 \mathrm{~K}$ in the relaxation strength for the $\alpha$ and $\beta$ processes, as shown in Figure 11(c), is the same phenomenon as the relaxation peak indicated by the arrow in Figure 13. The merging and splitting of both the $\alpha$ and $\beta$ relaxations are observed for the first time in our work. There are three ways to interpret the splitting of the $\alpha$ and $\beta$ processes [8]. Among them, the merged $\alpha$ and $\beta$ relaxations originate from the $\beta$ relaxation because the merged relaxation is described by the Arrhenius dependence of the $\beta$ relaxation (Figure 13), which is also found in the temperature dependence of the relaxation strength for the $\alpha$ and $\beta$ relaxations, as shown in Figure 11(c).

\section{Conclusions}

Dielectric relaxation spectroscopy was substantially employed to evaluate the dielectric response of a material. Polymer and polymer composites were subjected to dielectric relaxation spectroscopy for a wide range of times and temperatures to study the relaxation-related phenomena occurring between them. This dielectric spectroscopy was applied to two rubbery polymers to elucidate the relaxation processes with a self-developed dispersion analysis program. The analysis program decomposed the superposed dielectric spectra in the frequency domain, determined the relaxation processes, and made it possible to assign their peaks, thereby resulting in the characterization of entire relaxation processes.

In the NBR investigation results, five relaxation processes were identified, namely, $\alpha, \alpha^{\prime}, \beta, \gamma$, and conductivity relaxations, which could be explained in relation to chain structure and filler. The dielectric strength and shape parameters for the relaxation process were investigated as functions of temperature. The temperature behavior on the conductivity followed the Arrhenius equation at temperatures above the glass transition temperature. The activation energies for the $\alpha^{\prime}, \beta, \gamma$, and conductivity relaxations were obtained by applying the Arrhenius equation to the position of the imaginary permittivity peak versus the temperature. The VFT fits of the observed $\alpha$ relaxation were used to determine the glass transition temperature, and these values were compared with those obtained in a different study. The molecular chains responsible for the relaxation processes, $\alpha^{\prime}$ and $\alpha$, were also assigned by calculating the corresponding dipole moment through molecular modeling. 
In the EPDM investigation results, five relaxation processes were also identified: $\alpha, \beta, \alpha \beta$, MWS, and conductivity relaxations. The relaxation strength and shape parameter for these relaxation processes were obtained as a function of temperature, which presents information on relaxation. The temperature behavior on the conductivity relaxation followed an Arrhenius dependence. The activation energies for both the $\beta$, MWS and conduction relaxations were obtained by assuming Arrhenius behavior. The VFT fits of the observed $\alpha$ relaxation were used to determine the glass transition temperature. We discovered all the relaxations in the polymers by employing our developed dispersion analysis program. The merging and splitting of the $\alpha$ and $\beta$ relaxation peaks in the EPDM samples were observed for the first time in this work. We know that the $\alpha \beta$ relaxation originates from the $\beta$ process from the temperature dependence of the relaxation strength and relaxation peak. Therefore, dielectric relaxation spectroscopy can characterize the relaxation process and the related molecular dynamics in various polymers.

\section{Data Availability}

The data that support the findings of this study are available within the article.

\section{Conflicts of Interest}

The author(s) declare that there are no conflicts of interest regarding the publication of this paper.

\section{Acknowledgments}

This research was supported by the Development of Reliability Measurement Technology for Hydrogen Fueling Station funded by the Korea Research Institute of Standards and Science (KRISS-2020-GP2020-0007) and by the 2019 2021 KAIA/ MOLIT Project (no. 19TLRP-C152334-01) led by the Korea Research Institute of Standards and Science (KRISS).

\section{References}

[1] J. C. Yang, E. Hnetkovsky, D. Rinehart, M. Fernandez, F. Gonzalez, and J. Borowsky, "Performance of metal and polymeric O-ring seals during beyond-design-basis thermal conditions," Polymer Testing, vol. 58, pp. 135-141, 2017.

[2] R. R. Barth, K. L. Simmons, and C. S. Marchi, Polymers for Hydrogen Infrastructure and Vehicle Fuel Systems: Applications, Properties, and Gap Analysis, Office of Scientific and Technical Information (OSTI), Oak Ridge, TN, USA, 2013.

[3] S. Pehlivan-Davis, "Polymer electrolyte membrane (PEM) fuel cell seals durability," $\mathrm{Ph} \mathrm{D}$. Dissertation, Loughborough University, Loughborough, UK, 2015.

[4] N. C. Menon, A. M. Kruizenga, K. J. Alvine et al., Proceedings of the ASME 2016 Pressure Vessels and Piping Conference PVP 2016, Pressure Vessels and Piping Division, Vancouver, Canada, 2016.

[5] H. Fujiwara, H. Ono, and S. Nishimura, "Degradation behavior of acrylonitrile butadiene rubber after cyclic highpressure hydrogen exposure," International Journal of Hydrogen Energy, vol. 40, no. 4, pp. 2025-2034, 2015.
[6] S. Nishimura, International Symposium of Hydrogen Polymers Team, HYDROGENIUS, Shiiki Hall, Kyushu University, Fukuoka, Japan, 2017.

[7] W. G. Wang and X. Y. Li, "Impedance and dielectric relaxation spectroscopy studies on the calcium modified Na0.5Bi0.44Ca0.06TiO2.97 ceramics," AIP Advances, vol. 7, no. 12, Article ID 125318, 2017.

[8] J. P. Runt and J. J. Fitzgerald, Dielectric Spectroscopy of Polymeric Materials, American Chemical Society, Washington, DC, USA, 1997.

[9] F. Kremer and A. Schonhals, Broadband Dielectric Spectroscopy, Springer, Berlin, Germany, 2003.

[10] C. Ku and R. Liepins, Electrical Properties of Polymers, Hanser Publishers, Munich, Germany, 1987.

[11] C. Fernández-Sánchez, C. J. McNeil, and K. Rawson, “Electrochemical impedance spectroscopy studies of polymer degradation: application to biosensor development," TrAC Trends in Analytical Chemistry, vol. 24, no. 1, pp. 37-48, 2005.

[12] D. Dastan and A. Banpurkar, "Solution processable sol-gel derived Titania gate dielectric for organic field effect transistors," Journal of Materials Science: Materials in Electronics, vol. 28, no. 4, pp. 3851-3859, 2016.

[13] D. Dastan, S. W. Gosavi, and N. B. Chaure, "Studies on electrical properties of hybrid polymeric gate dielectrics for field effect transistors," Macromolecular Symposia, vol. 347, no. 1, pp. 81-86, 2015.

[14] G. Williams, "Dipole relaxation in polyethyl methacrylate and polyethyl acrylate as a function of frequency, temperature and pressure. The $\alpha, \beta$ and $\alpha \beta$ relaxations," Transactions of the Faraday Society, vol. 62, pp. 2091-2102, 1966.

[15] R. Brand, P. Lunkenheimer, U. Schneider, and A. Loidl, "Excess wing in the dielectric loss of glass-forming ethanol: a relaxation process," Physical Review B, vol. 62, no. 13, pp. 8878-8883, 2000.

[16] G. D. Smith and D. Bedrov, "Relationship between the $\alpha$ - and $\beta$-relaxation processes in amorphous polymers: insight from atomistic molecular dynamics simulations of 1,4-polybutadiene melts and blends," Journal of Polymer Science Part B: Polymer Physics, vol. 45, no. 6, pp. 627-643, 2007.

[17] D. Dastan, "Effect of preparation methods on the properties of titania nanoparticles: solvothermal versus sol-gel," Applied Physics A, vol. 123, no. 11, Article ID 699, 2017.

[18] J. Pietrasik, M. Gaca, M. Zaborski, L. Okrasa, G. Boiteux, and O. Gain, "Studies of molecular dynamics of carboxylated acrylonitrile-butadiene rubber composites containing in situ synthesized silica particles," European Polymer Journal, vol. 45, no. 12, pp. 3317-3325, 2009.

[19] J.-F. Chailan, G. Boiteux, J. Chauchard, B. Pinel, and G. Seytre, "Viscoelastic and dielectric study of thermally aged ethylenepropylene diene monomer (EPDM) compounds," Polymer Degradation and Stability, vol. 47, no. 3, pp. 397-403, 1995.

[20] F. Zhang, Q. Zhao, T. Liu, Y. Lei, and C. Chen, "Preparation and relaxation dynamics of ethylene-propylene-diene rubber/ clay nanocomposites with crosslinking interfacial design," Journal of Applied Polymer Science, vol. 135, no. 1, Article ID 45553, 2018.

[21] Y. Feldman, A. Puzenko, and Y. Ryabov, Dielectric Relaxation Phenomena in Complex Materials, John Wiley \& Sons, Inc., Hoboken, NJ, USA, 2006.

[22] E. Schlosser and A. Schönhals, "Recent development in dielectric relaxation spectroscopy of polymers," Colloid \& Polymer Science, vol. 267, no. 11, pp. 963-969, 1989.

[23] J. K. Jung, Y. I. Moon, K. S. Chung, and K.-T. Kim, “Development of a program for analyzing dielectric relaxation 
and its application to polymers: nitrile butadiene rubber," Macromolecular Research, vol. 28, no. 6, pp. 596-604, 2020.

[24] K. S. Cole and R. H. Cole, "Dispersion and absorption in dielectrics I. Alternating current characteristics," The Journal of Chemical Physics, vol. 9, no. 4, pp. 341-351, 1941.

[25] D. W. Davidson and R. H. Cole, "Dielectric relaxation in glycerol, propylene glycol, and n-propanol," The Journal of Chemical Physics, vol. 19, no. 12, pp. 1484-1490, 1951.

[26] S. Havriliak and S. Negami, "A complex plane analysis of $\alpha$-dispersions in some polymer systems," Journal of Polymer Science Part C: Polymer Symposia, vol. 14, no. 1, pp. 99-117, 2007.

[27] Polymer Properties Database, O-Ring Seals, 2015, https:// polymerdatabase.com/Elastomers/O-Ring_Elastomers.html.

[28] Polymer Properties Database, EPDM-Ethylene Propylene Diene Rubber, 2015, https://polymerdatabase.com/ Elastomers/EPDM.html.

[29] J. K. Jung, I. G. Kim, and K. T. Kim, "Evaluation of hydrogen permeation characteristics in rubbery polymers," Current Applied Physics, vol. 21, pp. 43-49, 2021.

[30] A. Schönhals, F. Kremer, A. Hofmann, E. W. Fischer, and E. Schlosser, "Anomalies in the scaling of the dielectric $\alpha$-relaxation," Physical Review Letters, vol. 70, no. 22, pp. 3459-3462, 1993.

[31] J. Langer, "The mysterious glass transition," Physics Today, vol. 60, no. 2, pp. 8-9, 2007.

[32] L. S. Garca-Coln, L. F. del Castillo, and P. Goldstein, "Theoretical basis for the Vogel-Fulcher-Tammann equation," Physical Review B, vol. 40, no. 10, pp. 7040-7044, 1989.

[33] H. Vogel, "Das temperaturabhängigkeitsgesetz der viskosität von flüssigkeiten," Physikalische Zeitschrift, vol. 22, pp. 645-646, 1921.

[34] G. S. Fulcher, "Analysis of recent measurements of the viscosity of glasses," Journal of the American Ceramic Society, vol. 8, no. 6, pp. 339-355, 1925.

[35] G. Tammann and W. Hesse, "Die abhängigkeit der viscosität von der temperatur bie unterkühlten flüssigkeiten," Zeitschrift für anorganische und allgemeine Chemie, vol. 156, no. 1, pp. 245-257, 1926.

[36] J. Menegotto, P. Demont, A. Bernes, and C. Lacabanne, "Combined dielectric spectroscopy and thermally stimulated currents studies of the secondary relaxation process in amorphous poly(ethylene terephthalate)," Journal of Polymer Science Part B: Polymer Physics, vol. 37, no. 24, pp. 3494-3503, 1999.

[37] S. Arrhenius, "Über die dissociationswärme und den einfluss der temperatur auf den dissociationsgrad der elektrolyte," Zeitschrift für Physikalische Chemie, vol. 4, no. 1, pp. 96-116, 1889.

[38] P. A. O'Connell and G. B. McKenna, "Arrhenius-type temperature dependence of the segmental relaxation below $T_{g}$," The Journal of Chemical Physics, vol. 110, no. 22, pp. 1105411060, 1999.

[39] M. Samet, V. Levchenko, G. Boiteux, G. Seytre, A. Kallel, and A. Serghei, "Electrode polarization vs. Maxwell-Wagner-Sillars interfacial polarization in dielectric spectra of materials: characteristic frequencies and scaling laws," The Journal of Chemical Physics, vol. 142, no. 19, Article ID 194703, 2015.

[40] X. Xia, Z. Zhong, and G. J. Weng, "Maxwell-Wagner-Sillars mechanism in the frequency dependence of electrical conductivity and dielectric permittivity of graphene-polymer nanocomposites," Mechanics of Materials, vol. 109, pp. 42-50, 2017.
[41] N. Paranjape, P. C. Mandadapu, G. Wu, and H. Lin, "Highlybranched cross-linked poly(ethylene oxide) with enhanced ionic conductivity," Polymer, vol. 111, pp. 1-8, 2017.

[42] M. Ribes, G. Taillades, and A. Pradel, "Non-Arrhenius conductivity in glassy and crystallized fast ion conductors: a manifestation of cationic disorder," Solid State Ionics, vol. 105, no. 1-4, pp. 159-165, 1998.

[43] W. Wieczorek, "Temperature dependence of conductivity of mixed-phase composite polymer solid electrolytes," Materials Science and Engineering: B, vol. 15, no. 2, pp. 108-114, 1992.

[44] C. J. F. Bottcher, Theory of Dielectric Polarization, Elsevier, Amsterdam, Netherlands, 1973.

[45] C. J. F. Bottcher and P. Bordewijk, Theory of Dielectric Polarization, Elsevier, Amsterdam, Netherlands, 1978.

[46] E. V. Bystritskaya, T. V. Monakhova, and V. B. Ivanov, “TGA application for optimising the accelerated aging conditions and predictions of thermal aging of rubber," Polymer Testing, vol. 32, no. 2, pp. 197-201, 2013. 